\title{
Preface
}

\section{Why Sleep Medicine Is \\ Essential}

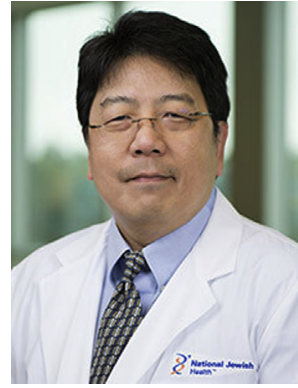

Teofilo Lee-Chiong, Jr, MD

Editor

While science continues to better understand the reasons why we sleep, such as physiologic drive and neurocognitive health, it is equally essential to recognize the mechanisms why we don't sleep. Three general reasons are responsible for this lack of sleep. We can't sleep due to sleep apnea, insomnia, or restless legs syndrome. We won't sleep as a result of social media and school or work schedules. Finally, we shouldn't sleep because of shift work or occupation as a commercial driver or pilot. Addressing these diverse factors requires a comprehensive approach that incorporates an understanding of anatomy and physiology, behavioral sciences and psychology, and public health and societal transformation.

We are increasingly more aware of how sleep affects our health. What is less certain is whether this knowledge leads to actions that optimize our sleep. Are we integrating better sleep habits into our general lifestyle? Are consumer-targeted wearables and mobile activity-monitoring technologies helping us achieve our desired sleep-worklife balance? Not infrequently, our efforts to adopt better sleep behaviors are thwarted by demands on our time by family, peers, school, or work. Having both personal and societal determinants, sleep optimization can only be attained if the community supports each individual's need for sleep, respects choices regarding sleep behaviors, and allocates enough time for adequate sleep for everyone. Corporations must develop strategies that foster sleep health, incorporating proper sleep behaviors among its employees and discouraging unnecessary after-work activities. Society at large also plays a major role in regulating work schedules of workers in more high-risk occupations, including pilots, public transportation crews, and hospital staff.

There is great global disparity in the availability of sleep health care, with many nations having well-established sleep medicine systems, yet others struggling to address the needs of their vulnerable populations with limited resources. For instance, it is estimated that nearly 425 million adults have moderate to severe obstructive sleep apnea globally today, and it is widely held that the majority remain either undiagnosed or untreated. Although we have accurate diagnostic tools and effective therapies for many sleep-related disorders, sleep testing and treatments are still unavailable for many, and adequate numbers of sleep health professionals are lacking in parts of the world. Primary care resources are essential but are, at present, underutilized. Incorporating sleep medicine into primary care can enhance efficiency and quality of care, increase access, and improve health outcomes.

All of us are eyewitnesses to, as well as participants of, an ongoing social transformation of sleep. Among the most primordial of physiologic 
drives, sleep, today, is being reshaped by lifestyle, technology, pharmacology, and policy. This rapidly evolving part of human behavior and communal life has a wide-ranging impact on personal health, relationships, performance, and public safety. For many, adequacy of sleep is deemed both necessary yet unattainable, and solutions for sleep disorders are considered effective but remain inaccessible or unaffordable. As we continue to imagine what our sleep should or could be, it is essential we remember that sleeping is not an evolutionary error of physiology or simply a momentary pause in a highly scheduled calendar; rather, it exists side by side with waking life, and neither endures without the other.

Teofilo Lee-Chiong, Jr, MD National Jewish Health Denver, CO 80206, USA

University of Colorado School of Medicine Aurora, CO 80045, USA

E-mail address: Lee-ChiongT@njc.org 